\title{
Clinico-Radiological and Functional Outcome of Precontoured Lateral Clavicle Locking Compression Plate with Broad Lateral End in Lateral End Clavicular Fractures
}

ISSN: 2576-8875

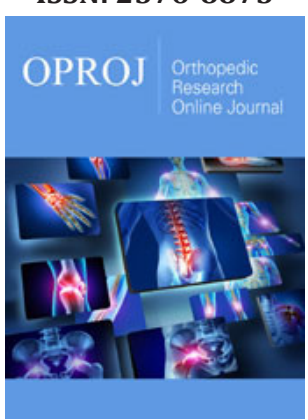

${ }^{* 1}$ Corresponding author: Ashwin Gobbur, Department of Orthopaedics, SS Institute of Medical Sciences and Research, Davangere, Karnataka, India-577004

Submission:

Published: 侮 October 11, 2019

Volume 6 - Issue 1

How to cite this article: Venkataramana R, Srinath SR, Manjunath J, Sachin N, Ashwin G. Clinico-Radiological and Functional Outcome of Precontoured Lateral Clavicle Locking Compression Plate with Broad Lateral End in Lateral End Clavicular Fractures. Ortho Res Online J. 6(1). OPROJ.000628.2019.

DOI: 10.31031/OPROJ.2019.06.000628

Copyright@: Ashwin Gobbur, This article is distributed under the terms of the Creative Commons Attribution 4.0 International License, which permits unrestricted use and redistribution provided that the original author and source are credited.
Venkataramana RM, Srinath SR, Manjunath J, Sachin Nimbaragi and Ashwin Gobbur*

Department of Orthopedics, SS Institute of Medical Sciences and Research, Davangere, Karnataka, India

\begin{abstract}
Background: Lateral end clavicle fractures account for 12 to $15 \%$ of clavicle fractures [1]. With various recommendations for treating fracture of lateral end of the clavicle, unfortunately there is no reference standard treatment recommendation for this fracture. The unstable nature of these fractures makes them more prone for non-union and impeding the normal shoulder function. Recently, precontoured clavicular locking compression plate with broad lateral end has been introduced that enables stable angular fixation of distal fragment regardless of bone quality, reduces the risk of above foresaid complications.
\end{abstract}

Objectives: To evaluate the clinico-radiological and functional outcomes of lateral end clavicular fractures using precontoured lateral clavicle locking compression plate with broad lateral end.

Materials and methods: After screening of patients, a total of 79 cases, who were enrolled in the study, were subjected for surgical management of lateral end clavicular fractures with precontoured lateral clavicle locking plate. All the patients were followed up clinically and radiologically at the immediate post op period and at the end of 1, 3, 6, 12 and 18 months. The efficacy and functional outcome of precontoured lateral clavicle locking plate were charted according to DASH scores. All cases were followed up for complications and were treated accordingly.

Results: In our study, 79 cases underwent surgical management with precontoured lateral clavicle locking plate. The results were analysed according to DASH scores showed excellent in 43 cases (54.43\%), good in 32 cases $(40.50 \%)$ and poor in 4 cases $(5.06 \%)$. The correlation analysis with Pearson's correlation coefficient (r) was 0.712 which show highly positive correlation between union of lateral end clavicular fracture and precontoured lateral clavicle locking plate.

Conclusion: We recommend the usage of precontoured clavicular locking compression plate with broad lateral end enables stable angular fixation of distal fragment regardless of bone quality and reduces the risk of complications.

Keywords: Lateral end clavicular fractures; Precontoured lateral clavicle locking plate; DASH score

\section{Introduction}

Clavicle is the only long bone lying horizontally in the body. Lateral end clavicle fractures account for 12 to $15 \%$ of clavicle fractures [1]. With various recommendations for treating fracture of lateral end of the clavicle, unfortunately there is no reference standard treatment recommendation for this fracture [2]. The unstable nature of these fractures makes them more prone for non-union and impeding the normal shoulder function. The rate of non-union in lateral end clavicular fractures can be as high as 22 to $44 \%$ because of strong displacing force between weight of the arm distracting the distal fragment and counter-pull of trapezius on the proximal fragment $[3,4]$.

Various treatment modalities are available such as trans- or extra-articular K wire fixation [4], Knowles pin fixation [5], tension band wire fixation [6,7], and coracoclavicular screw fixation [8]. The fixation methods are associated with various complications like pin migration, degeneration of acromio-clavicular joint, loss of reduction, penetration of screw through the bone and skin ulceration because of pin irritation [9]. Furthermore, rigid fixation and early mobilization is difficult after the above-mentioned methods because of small comminuted soft meta-physeal distal bony fragment which does not provide the stable fixation construct 
[10]. Some surgeons used the hooked plate with an extension under the acromion which led to the development of rotator cuff injury, sub-acromial impingement or bursitis, clavicular stress fracture at medial end of plate and need for early removal of plate $[11,12]$.

Recently, precontoured clavicular locking compression plate with broad lateral end has been introduced that enables stable angular fixation of distal fragment regardless of bone quality, reduces the risk of above foresaid complications [13]. This study was performed to evaluate the clinico-radiological and functional outcomes of lateral end clavicular fractures using precontoured lateral clavicle locking compression plate with broad lateral end.

\section{Materials and Methods}

With a level IV evidence, a prospective cohort study was performed from 2016 to 2018 in the department of Orthopaedics, SS Institute of Medical Sciences and Research, Davangere, Karnataka, India. The cases for this study were recruited by convenient sampling technique. Among 119 clinically and radiologically confirmed lateral end clavicular fracture cases, a total of 27 cases were excluded (failed to satisfy inclusion criteria $n=12$ and decline to participate $n=15$ ) and remaining 92 cases underwent surgical management as per our study protocol. A total of 13 cases lost follow up after surgical procedure in our study period. Hence 79 cases were taken into consideration for statistical analysis.

The patients with age between 20 to 70 years of age, patients with clinically and radiologically confirmed lateral end clavicular fractures (Neer's types 2) and patients with type 1, 2, 3a \& 3b compound femoral fractures according to Gustilo Anderson classification for open fractures were included in the study. The patients with age less than 20 and more than 70 years of age, patients with medial $1 / 3^{\text {rd }}$ and middle $1 / 3^{\text {rd }}$ clavicular fractures, patients with type $3 \mathrm{c}$ compound femoral fractures according to Gustilo Anderson classification for open fractures, patients with lateral end clavicular fractures of more than 7 days old and patients with pathological fractures over lateral end of clavicle were excluded from the study (Figure 1).
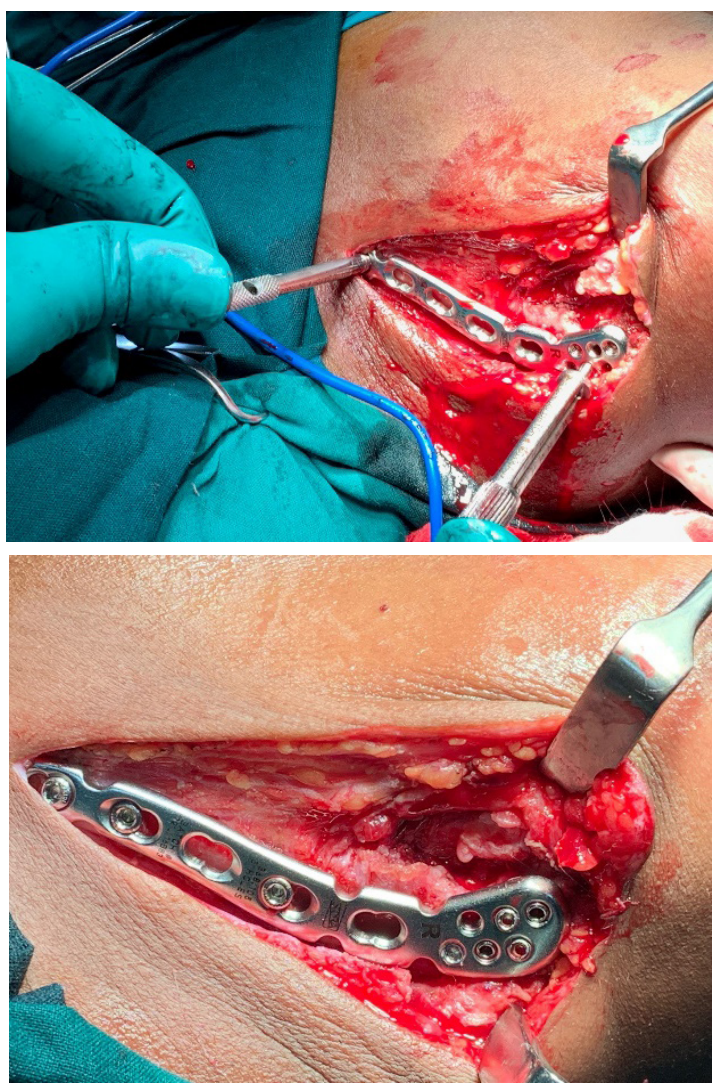

Figure 1: Lateral end clavicular fractures fixed with precontoured clavicular locking compression plate with broad lateral end.

All the patients enrolled in the study were subjected for thorough clinical examination. The baseline investigations and radiographic analysis of the affected clavicle were analysed. According to Gustilo and Anderson classification of open fractures, the compound fractures were treated with wound debridement and IV antibiotics in view to control any infection. All the cases were posted for surgical management with precontoured lateral clavicle locking plate and follow up according to our study protocol.
The radiological assessment was done with AP and axial views of shoulder with clavicle. The functional outcome of fractures was assessed by DASH scores. The follow up of cases were done with regular clinical and radiological analysis at the immediate post op period and at the end of 1, 3,6,12 and 18 months. All the patients were offered implant removal at an average of 18 months postsurgical procedure who showed clinical and radiological fracture union. 


\section{Results}

A total of 92 cases of lateral end clavicular fractures, who were enrolled in the study, were treated with precontoured lateral clavicle locking plate. A total of 13 cases lost follow up post procedure. Hence 79 cases were taken into consideration for statistical analysis. The descriptive statistics were reported as mean (SD) for continuous variables, frequencies (percentage) for categorical variables. Data were evaluated with IBM SPSS Statistics for Windows, Version 20.0, IBM Corp, Chicago, IL.

Table 1: Patient's demography.

\begin{tabular}{|c|c|c|c|}
\hline Age (years) & Males & Females & Total \\
\hline $11-25$ & 12 & 5 & $17(21.51 \%)$ \\
\hline $26-40$ & 23 & 17 & $40(50.63 \%)$ \\
\hline $41-55$ & 8 & 6 & $14(17.72 \%)$ \\
\hline $56-70$ & 3 & 5 & $8(10.12 \%)$ \\
\hline
\end{tabular}

Among 79 cases, 46 cases (58.22\%) were males and 33 cases $(41.77 \%)$ were females. The maximum number of cases belong to $26-40$ years age group i.e 40 cases (50.63\%) followed by 11-25 years age group i.e 17 cases $(21.51 \%)$. The mean age of cases in the study was $37.28 \pm 5.72$ years (Table 1 ).

Among 79 cases, 42 cases (53.16\%) sustained injury due to road traffic accident, 21 cases (26.58\%) fall from height and 16 cases $(20.25 \%)$ due to trivial fall. There was no patient reported with bilateral clavicular fractures. All the patients were clinically and radiologically confirmed lateral end clavicular fractures (Neer's types 2). According to Gustilo Anderson classification, type 1 compound cases were managed with tetanus toxoid and IV antibiotics pre-operatively. Type 2 , 3a and 3 b compound cases were managed with tetanus toxoid, IV antibiotic coverage and a thorough wound debridement. All cases underwent surgery with an average of $2.73 \pm 0.39$ days. The mean duration of fracture union was $18.47 \pm 2.01$ weeks. All the cases were followed up serially as per our protocol with serial clinical and radiographical examinations (Figure 2-4).

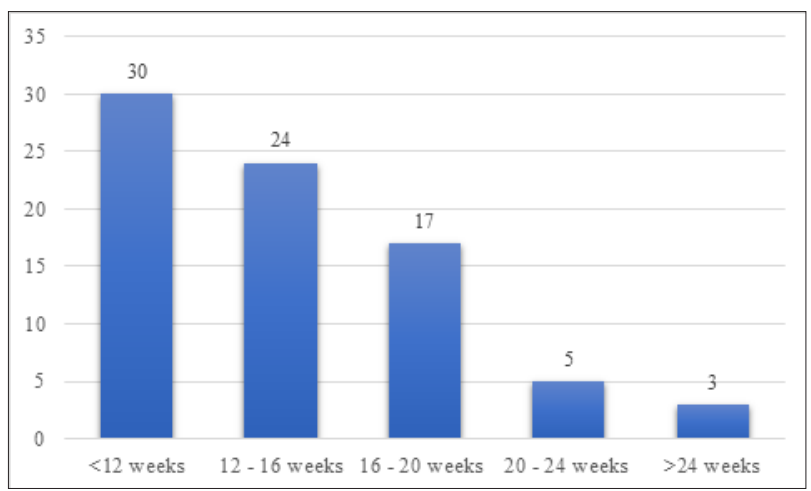

Figure 2: Duration of fracture union.

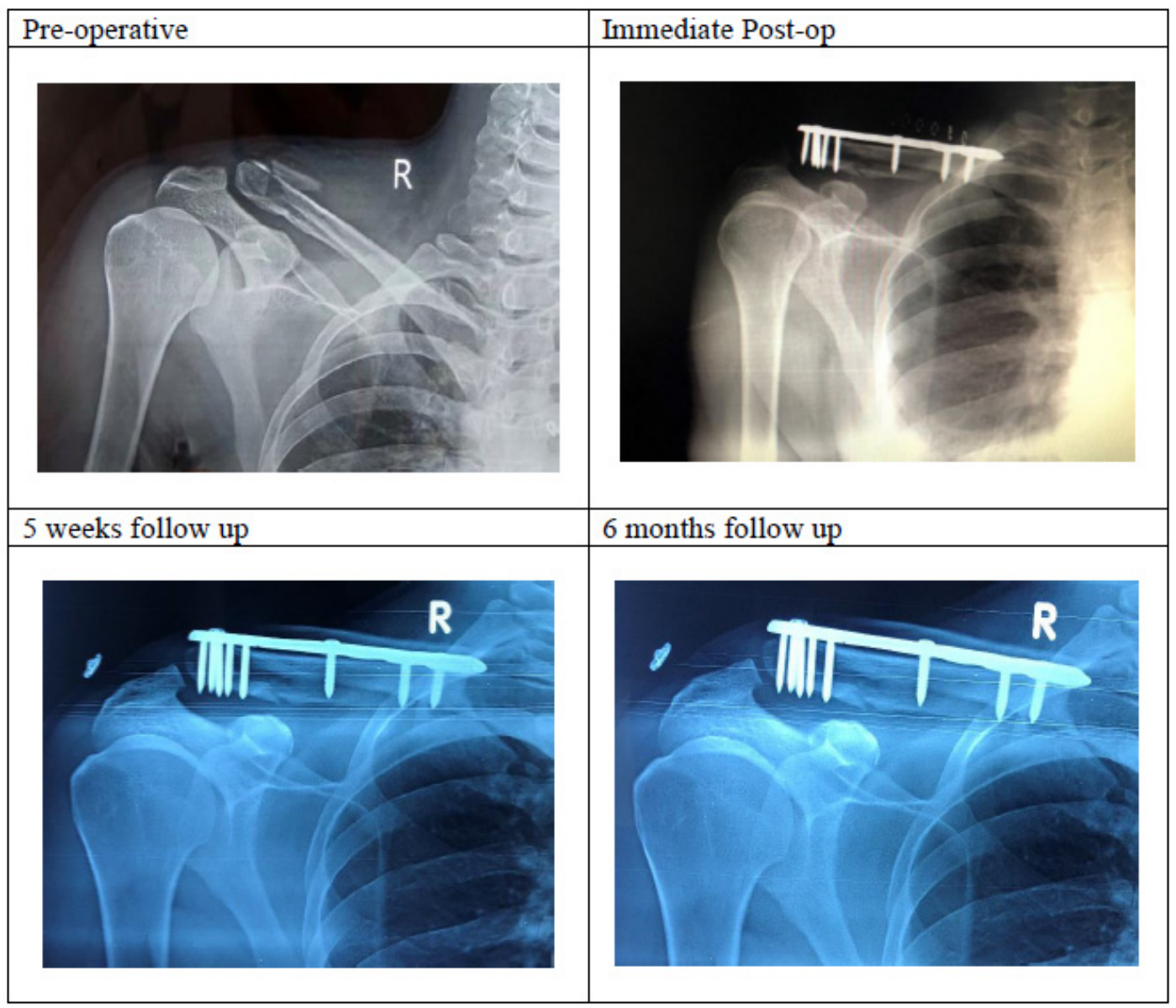

Figure 3: Radiographs of precontoured lateral clavicle locking compression plate with broad lateral end. 


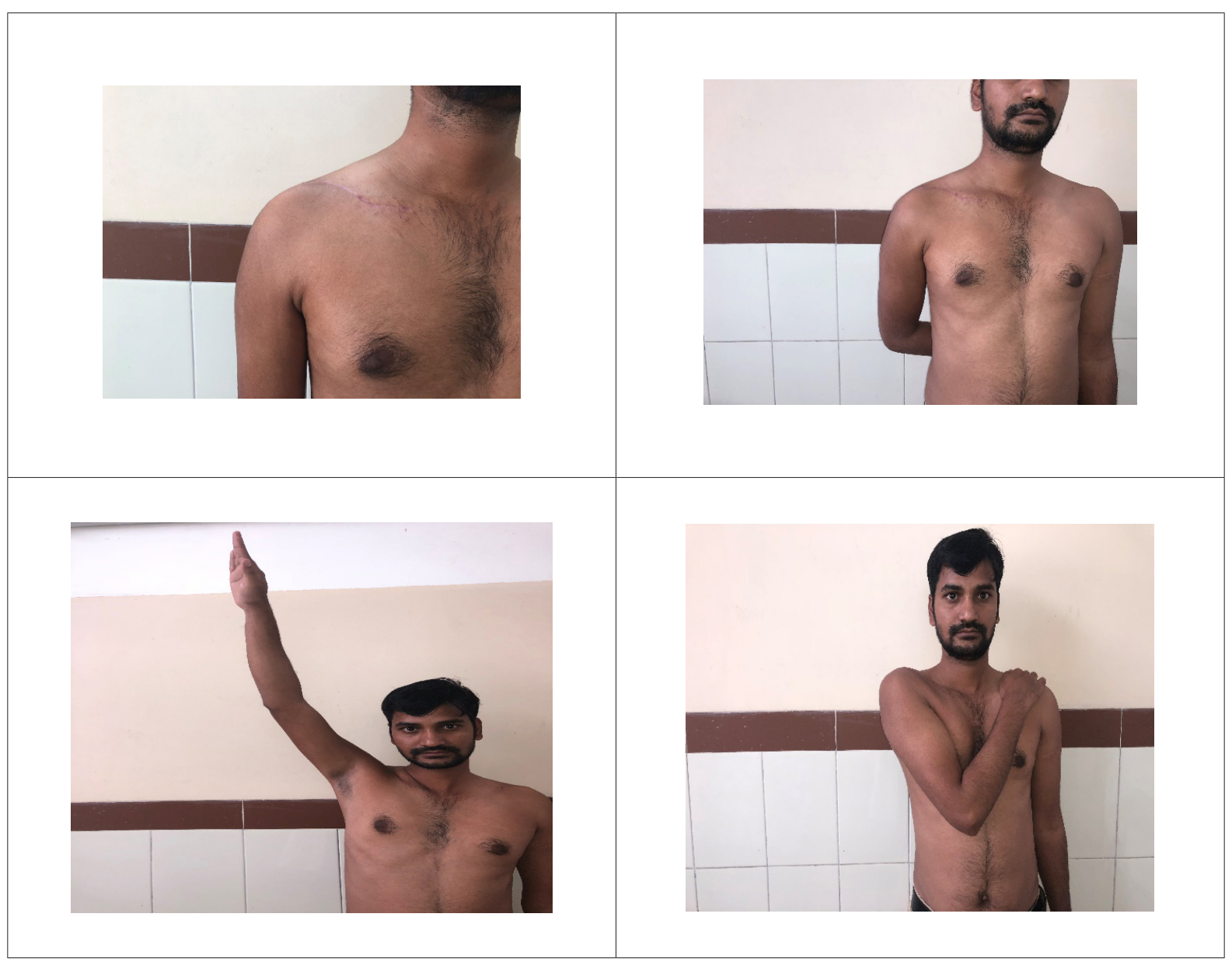

Figure 4: Clinical outcome in patient with precontoured lateral clavicle locking compression plate with broad lateral end at 18 months post-surgical procedure.

Out of 79 cases, at the end of 1 year follow up, 64 cases $(73.45 \%)$ achieved full range of shoulder movements. The mean range of movements at the end of 1 year were $130^{\circ}$ flexion, $50^{\circ}$ extension, $160^{\circ}$ abduction, $80^{\circ}$ adduction, $60^{\circ}$ internal rotation and $70^{\circ}$ external rotation. The remaining 15 cases with reduced range of shoulder movements were treated with physiotherapy in the form active and passive pendulum, ladder and wheel exercises.

The most common complication encountered in our study was malunion 4 cases $(5.06 \%)$. No cases of infection, implant breakage, non-union and neurovascular deficit were reported in our study. The results were analysed according to DASH scores showed excellent in 43 cases (54.43\%), good in 32 cases (40.50\%) and poor in 4 cases $(5.06 \%)$. The poor range of movements $(n=4)$ were due to malunion of fracture. The correlation analysis with Pearson's correlation coefficient (r) was 0.712 which show highly positive correlation between union of lateral end clavicular fracture and precontoured lateral clavicle locking compression plate.

\section{Discussion}

The lateral end clavicular fractures account for 3\% of fractures of the clavicle yet they pose a significant morbidity in a significant number of patients $[2,3]$. There is a high rate of non-union which account for half of all clavicular non-unions when treated nonoperatively. Non-operative management are associated with poor outcomes in the form of malunion and non-union. A numerous method of surgical treatment of lateral end clavicular fractures have been described in the literature but there is no standard surgical modality for treating these fractures [5-12].

Mani et al. [13] have used precontoured clavicular locking plate with broad lateral end in 46 patients with distal third clavicle fractures. They reported the mean Constant-Murley score was $92.56 \pm 4.47$ for injured side with p-Value 0.56 and the mean UCLA Shoulder Rating Score was $32.55 \pm 2.12$ for injured side with $p$ value 0.58 . They concluded that precontoured clavicular locking plate provided the multi-planar fixation and greater stability for small unstable lateral fragment.

Anderson et al. [14] concluded LCP for lateral end clavicular plating was associated with high union rates, excellent functional outcomes and low complications rates in a case series of 13 patients with lateral end clavicular fractures. Klein et al. [15] compared the functional outcomes between the precontoured locking plates with suture augmentation in 16 patients and clavicular hook plates in 22 
patients with lateral clavicular fractures. They found early fracture union in both group and those with precontoured locking plates resulted with the superior functional results.

Chunline et al. [16] performed a comparative study between the locking compression plates in 36 patients and hook plates in 30 patients and found that functional outcomes were better in the group of locking plates. Sambandam et al. [17] concluded that clinical outcomes were equal in clavicular fractures fixed by flexible fixation like K-wires and tension banding wiring and rigid fixation by locking plates. However, they also noted that it was easy to remove the flexible implants under local anaesthesia while rigid implants required general anaesthesia for their removal.

Here in this article, we have used precontoured lateral clavicle locking plate in lateral end clavicular fractures to study the clinic-radiological and functional outcome of these fractures. We encountered the most common complication in our study as malunion 4 cases $(5.06 \%)$ without any other cases of infection, implant breakage, non-union and neurovascular deficit. The results were analysed according to DASH scores showed excellent in 43 cases $(54.43 \%)$, good in 32 cases $(40.50 \%)$ and poor in 4 cases $(5.06 \%)$. The poor range of movements $(n=4)$ were due to malunion of fracture. We obtained a highly positive Pearson's correlation $(r=0.712)$ between union of lateral end clavicular fracture and precontoured lateral clavicle locking compression plate.

\section{Conclusion}

Due to decreased morbidity and less foreseen complication associated in these patients, we recommend the usage of precontoured clavicular locking compression plate with broad lateral end in Neer's type II fractures. These plates enabled stable angular and multi-planar fixation of distal fragment regardless of bone quality, biomechanically stable and provided good results in the fracture fixation of the small distal fragments of the lateral end clavicular fractures.

\section{References}

1. Vaishya R, Vijay V, Khanna V (2017) Outcome of distal end clavicle fractures treated with locking plates. Chin J Traumatol 20(1): 45-48.

2. Moore TO (1951) Internal pin fixation of fracture of the clavicle. Am Surg 17: 580-583.
3. Bosworth BM (1941) Acromio-clavicular separation: new method of repair. Surg Gynecol Obstet 73: 866.

4. Constant CR, Murley AHG (1987) A clinical method of functional assessment of the shoulder. Clin Orthop Related Res 214: 160-164.

5. Levy 0 (2003) Simple, minimally invasive surgical technique for treatment of type 2 fractures of the distal clavicle. J Shoulder Elbow Surg 12(1): 24-28.

6. Fann CY, Chiu FY, Chuang TY, Chen CM, Chen TH (2004) Trans-acromial Knowles pin in the treatment of Neer type 2 distal clavicle fractures: A prospective evaluation of 32 cases. J Trauma 56(5): 1102-1105.

7. Shin SJ, Roh KJ, Kim J0, Sohn HS (2009) Treatment of unstable distal clavicle fractures using two suture anchors and suture tension bands. Injury 40(12): 1308-1312.

8. Bezer M, Aydin N, Guven O (2005) The treatment of distal clavicle fractures with coracoclavicular ligament disruption: a report of 10 cases. J Orthop Trauma 19(8): 524-528.

9. Macheras G, Kateros KT, Savvidou OD, Sofianos J, Fawzy EA, et al. (2005) Coracoclavicular screw fixation for unstable distal clavicle fractures. Orthopedics 28(7): 693-696.

10. Flinkkila T, Ristiniemi J, Lakovaara M, Hyvonen P, Leppilahti J (2006) Hook-plate fixation of unstable lateral clavicle fractures: a report on 63 patients. Acta Orthop 77(4): 644-649.

11. Kashii M, Inui H, Yamamoto K (2006) Surgical treatment of distal clavicle fractures using the clavicular hook plate. Clin Orthop Relat Res 447: 158164.

12. Haidar SG, Krishnan KM, Deshmukh SC (2006) Hook plate fixation for type II fractures of the lateral end of the clavicle. J Shoulder Elbow Surg 15(4): 419-423.

13. Kapil-Mani KC, Acharya P, Arun S (2018) Precontoured clavicular locking plate with broad lateral end: A newly designed plate for lateral third clavicle fractures. Malaysian Orthopaedic Journal 12(1): 15-20.

14. Anderson K (2003) Evaluation and treatment of distal clavicle fractures. Clin Sports Med 22: 319-326.

15. Klein SM, Badman BL, Keating CJ, Devinney DS, Frankle MA, et al. (2010) Results of surgical treatment for unstable distal clavicular fractures. J Shoulder Elb Surg 19: 1049-1055.

16. Chunlin Z, Junwu H, Yi L (2014) Comparison of the efficacy of a distal clavicular locking plate versus a clavicular hook plate in the treatment of unstable distalclavicle fractures and a systematic literature review. Int Orthop 38: 1461-1468.

17. Sambandam B, Gupta R, Kumar S, Maini L (2014) Fracture of distal end clavicle: A review. J Clin Orthop Trauma 5(2): 65-73. 